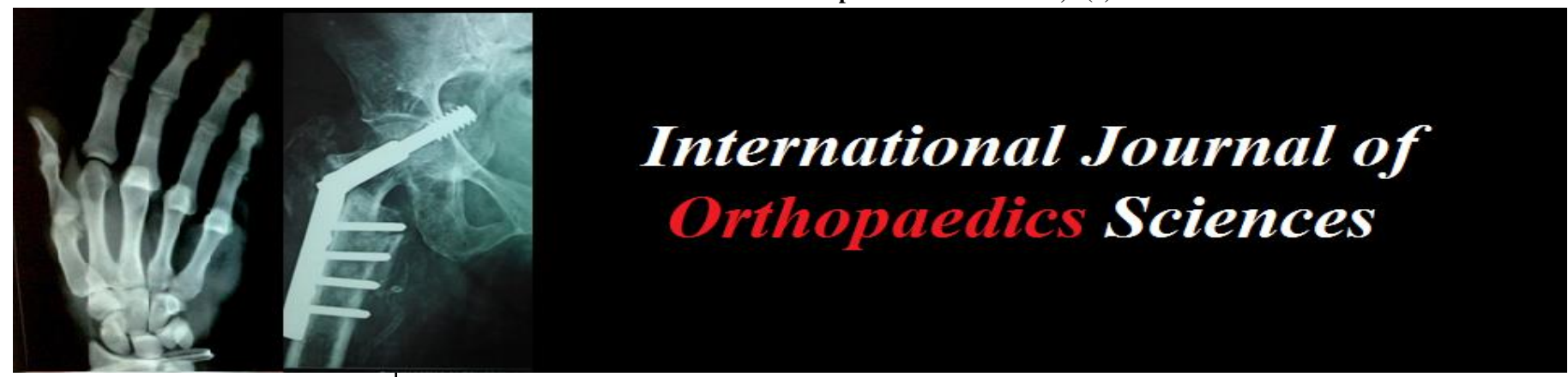

ISSN: $2395-1958$

IJOS 2018; 4(2): 802-806

(C) 2018 IJOS

www.orthopaper.com

Received: 25-02-2018

Accepted: 26-03-2018

V Vijayaraj

Prof., M.P.T., Ph.D, Research Scholar, HoD, Department of Neurology, Nandha College of Physiotherapy, Erode,

Vailkaalmedu, Tamil Nadu, India
Correspondence

V Vijayaraj

Prof., M.P.T., Ph.D, Research Scholar, HoD, Department of Neurology, Nandha College of Physiotherapy, Erode,

Vailkaalmedu, Tamil Nadu, India

\section{A comparative study between McKenzie technique and neural mobilization in chronic low back pain patients with radiculopathy}

\section{Vijayaraj}

DOI: https://doi.org/10.22271/ortho.2018.v4.i21.115

\section{Abstract}

Purpose: To study the effectiveness of McKenzie Technique and Neural Mobilization in chronic low back with radiculopathy on pain, spinal mobility and disability.

Relevance: Radiculopathy also known as nerve root pain which arise from disc herniation or spinal stenosis or post-operative scarring, it radiates down the leg in a dermatomal pattern, the unilateral leg pain is often described by the patient as worse than the back pain. McKenzie technique and neural mobilization techniques are the forms of manual therapy that are used in an effort to reduce radiating pain and improve range of motion. Efficient and effective management of acute low back pain is necessary hence to study the effectiveness of either technique in managing chronic low back with radiculopathy was the objective of this research report.

Participants: 30 subjects with chronic low back with radiculopathy were recruited from Nandha College of Physiotherapy, Erode, Government Head Quarters Hospital, Erode L.K.M Hospital, Erode. Methods: Subjects were randomly grouped into 2 group's viz. Group A (TENS with traction and McKenzie technique) \& Group B (TENS with traction and neural mobilization technique). The outcome measures were visual analogue scale (VAS) and disability level in terms of Modified Oswestry Disability Questionnaire (MODQ). Results: Within group's comparison showed significant result for Pain and MODQ score and between group's comparison showed significant result for Pain MODQ score respectively indicating effectiveness of McKenzie technique

Conclusions: The results of this study showed that along with TENS + Traction, McKenzie Technique is significant in decreasing pain, improving functional ability and increasing spinal extension in chronic low back with radiculopathy.

Keywords: Chronic low back pain, McKenzie technique, neural mobilization technique, visual analoque scale, modified oswestry disability questionnaire

\section{Introduction}

Low back Ache is an ache or discomfort in area of lower part of the back and spinal column it is characterized by a range of symptoms which includes pain muscle tension or stiffness and is localized below the costal margin and above the inferior gluteal folds with or without sciatic involvement and it is defined as chronic when it presists for 12 weeks or more.

It causes variety of diseases and disorders that affect lumbar spine this problem is attributed to the inability to control the deep muscle such as transverse abdominus and the multifidus that play important roles in maintaining spinal stability and damage proprioceptive sensory tissue of the lumbar spine and trunk. Low back pain may affect motor control of trunk muscle that regulates spinal movements and ability. The intervertebral disc is the common cause of back pain and the most common cause of radiculopathy.

Radiculopathy also known as nerve root pain which arise from disc herniation with nerve root compression. Nerve irritation caused by damage to the disc between the vertebrae damage to the disc occurs because of degeneration [wear and tear] of the outer ring of the disc traumatic injury or both a result the central softer portion of the disc can rupture (herniation) through the outer ring of the disc and about the spinal cords or its nerves as they exit the bony spinal column this rupture is what causes the commonly recognized pain of sciatica" that shoots down the leg. 


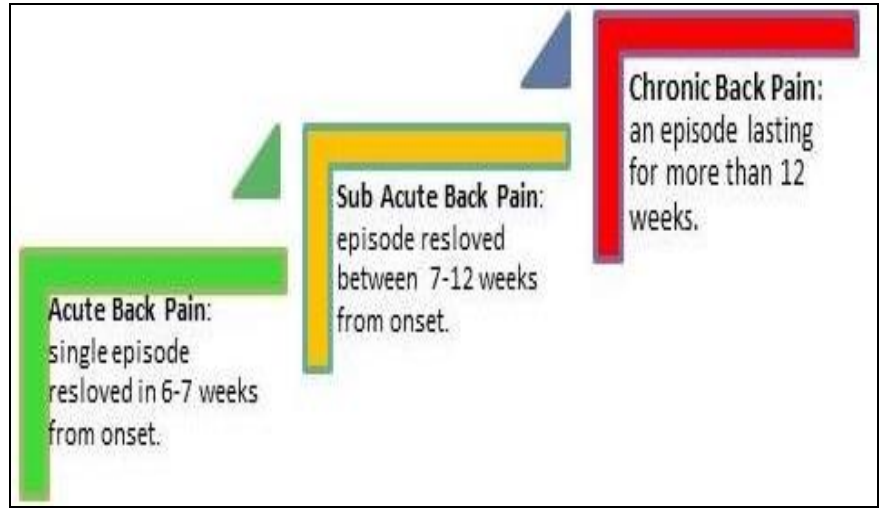

\section{Need For the Study}

Most of the population are suffering from back pain at some point that interferes with their Job. day by day activities and recreation. Back pain affects both physical ability as well as psychosocial health.

Both sexes are equally affected between the ages of 25 and 60 by low back pain.

There are various treatment available to treat low back pain, though various method of exercises are available, with the focus of better treatment. This study would like to find out the efficiency of McKenzie technique exercise and neural mobilization in radiculopathy low back pain improving functional ability and increasing spinal extension in chronic Low Back ache.

\section{Aim of the Study}

The purpose of the study is to find out the effects of McKenzie Technique and Neural

Mobilization on pain, and functional ability in patients with Chronic LBA

\section{Materials and Methodology \\ Materials}

- Treatment Couch

- Pillows

- Blankets

- Stethoscope

- $\quad$ B.P Apparatus

- Traction Machine

- Traction belt

- Foot Stool

- TENS

- Electrodes

- Cable

- Gel

- Cotton/Tissue papers

\section{Methodology}

- All patients underwent a Orthopaedic examination and posture evaluation.

- $\quad$ The Slump test, Faber's test, Bilateral straight leg raising test, and Prone lumbar instability test is conducted to confirm the diagnose of Chronic LBA

- VAS is conducted to know the severity of Pain in patients with Chronic Low Back Ache.

- MODQ is conducted to know the severity of the Disability in patients with Chronic Low back Ache.

\section{Population}

Patient with Chronic Low Back Ache with Radiculopathy who were between 25-60 year considered as population.

\section{Inclusion Criteria}

- A patient with chronic low back pain with radiculopathy

- Age 25-60 years

- Both sexes

- Centralization phenomenon, determined by using active movements testing has to be presents

- Symptoms more than 3 months

\section{Exclusion Criteria}

- Inflammatary

- Infection

- Metabolic disorders of spine

- Maligancy

- History of vertebral fracture

- Current pregnancy

- History of spinal surgery

- Patient with neurological deficit

- Patient with cardiovascular involvement

- Psychological pain

- Morbid obesity

- Structural deformity on spine

- Osteoporosis

- Spondylolisthesis

- Unco-operative patients

\section{Source of Data}

- Nandha College of Physiotherapy, Erode

- Government Head Quarters Hospital, Erode

- $\quad$ L.K.M Hospital, Erode.

\section{Samble Size}

- Sample size is 30 subjects

- Group A-15 patients

- Group B-15 patients

\section{Study Design}

Pre and Post experimental Study Design

Sampling Method

Convenient Sampling Method

Variables of the study

Independent variables

1. McKenzie Technique.

2. Neural Mobilization.

Dependent Variable

1. Visual Analogue Scale.

2. Modified Oswesry Disability Questionnaire.

\section{Duration of the Study}

6 Months

\section{Treatment Duration}

- Study was carried out for 4 weeks for each individual.

- McKenzie Technique was performed 20 minutes session five days per week.

- Neural Mobilization was performed 3 minutes on/off 3 repetition session five days per week.

- Both group participants received treatment of TENS for 15 minutes and traction for 20 minutes. 


\section{Parameter}

(a) Visual Analogue Scale

(b) Modified Oswestry Disability Questionnaire.

\section{Procedure}

Subjects who are diagnosed with chronic low back pain with radiculopathy were required for this study. A clear explain about the study was given to all the patients and they were included following suitable inclusion and exclusion criteria. An informed consent was obtained from all the participants and they were allowed to withdraw from the study at any point of time in case of any discomfort. After obtaining consent from all subjects then completed a through physical examination follwing the assessment patients values were assessed using Visual Analog Scale and Modified Oswestry Disability Index. 30 subjects with chronic Low Back Ache were selected and divided into 2 groups, with 15 in each groups.

\section{Group A: (McKenzie technique)}

They received McKenzie exercise to the back.

The participants received treatment of TENS for 15 minutes and traction for 20 minutes followed by McKenzie technique.

\section{McKenzie technique \\ Step-1}

Lie face down on an exercise mat. Place hands on either side of patients hand and forehead on the floor. If this position is uncomfortable, place a small pillow beneath abdomen to lesion the dress on lower back. Remain in this position for 5 minutes.

\section{Step-2}

From this position move to next position, rise up on to elbow and place your forearms flat on the floor. Lift chin slightly and hold this position for a further 5 minutes.

\section{Step-3}

Place hands beneath shoulder and keeping hips on the floor, rise chest off the ground by pressing with arms. Gradually increase range of movement as the set progress. On completion place a pillow beneath chest and relaxed in extended position.

\section{Step-4}

After a few movements add another pillow to further increase spinal extension. If it is still comfortable, add a third and final pillow and then hold this extended position for up to 10 minutes.

\section{Group B: (Neural mobilization)}

They received neural mobilization to the back.

The patient received treatment of TENS for 15 minutes and traction for 20 minutes followed by technique.

Neural mobilization: The patient is in supine lying and the leg was lifted upward as a solid lever, while maintaining the knee extension. The leg was raised past 35 degree in order to take up the slack in the nerve.

Sciatic nerve is completely stretched at 70 degree. For additional sensitization hip adduction was added to straight leg rising. The intervention consists of gentle short duration and large amplitude passive movements were perform at 'feather edge, of the patient neural symptoms in on/off fashion.

A mild degree of discomfort was permitted during on phase which must be completely abate when the tension was withdrawn (off phase). 3 minutes of on/off mobilization of 3 repetition was performed.
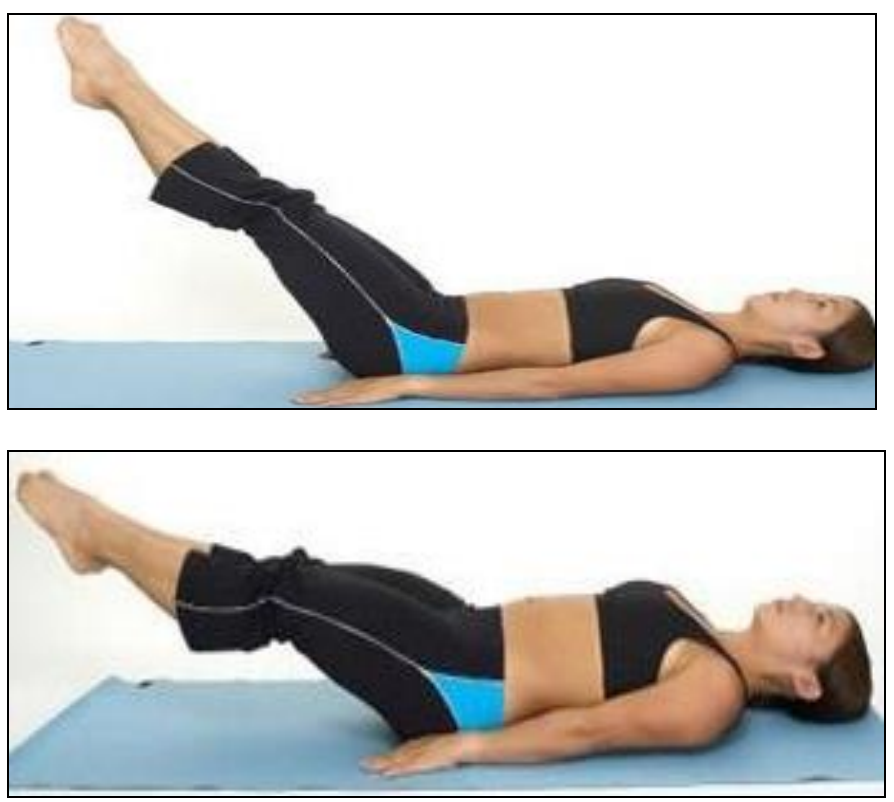

Leg raised 35 degree and 75 degree

\section{Chapter-IV}

Data Presentation and Stastistical Analysis

\section{Statistical Tools}

The paired t-test was used to find out the statistical significance between Pre and Post t-test values of VAS and MODQ Before and after treatment for Group A and Group B, and also unpaired t test is used to find out the significance in between two groups.

Mean Difference, Standard Deveiation and Paired't' Test Value between Group A and Group B of Vas and Modq

\begin{tabular}{|c|c|c|c|c|c|c|c|}
\hline \multirow{2}{*}{ Groups } & \multicolumn{2}{|c|}{ Mean difference } & \multicolumn{2}{c|}{ Standard deviation } & \multicolumn{2}{c|}{ Calculated 't' value } & \multirow{2}{*}{ Significance } \\
\cline { 2 - 8 } & Vas & Modq & Vas & Modq & Vas & Modq & \\
\hline Group a & 8 & 7 & 1.12 & 2 & 10.16 & 13.3 & Significant \\
\hline Group b & 2 & 2 & 1.06 & & 7.6 & 12.06 & Significant \\
\hline
\end{tabular}

Comparision of Unpaired ' $T$ ' Test and Table Value between Vas and Modq

\begin{tabular}{|c|c|c|c|}
\hline Parameters & Unpaired ' $\mathbf{t}$ ' Test & Table value & Significance \\
\hline Vas & 15 & 2.05 & Significant \\
\hline Modi & 14.28 & 2.05 & Significant \\
\hline
\end{tabular}


Comparision of Unpaired ' $T$ ' Test and Table Value Between vas and modq

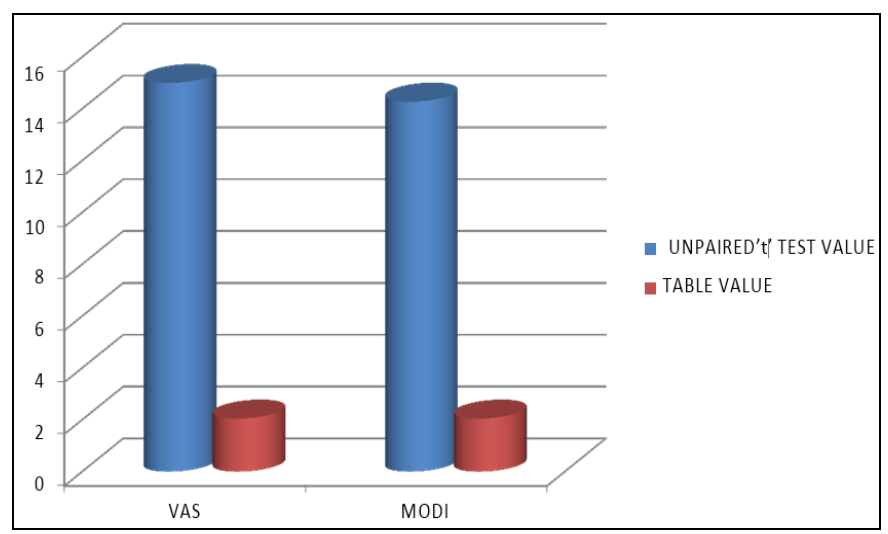

\section{Result and discussion \\ Results}

The study sample comprised 30 patients, of which 15 were group A and 15 were group B. The diagnostic test for Chronic Low Back Ache with radiculopathy was positive in 30 patients. The median time interval between VAS and MODQ Questionnaires applied before and after therapy was 4 weeks. Among 30 patients, 15 were treated with McKenzie technique, and 15 were treated with neural mobilization

The pre and posttest values were assessed by VAS and MODQ in was tabulated above and the paired test values have shows that the McKenzie Technique was more effective than neural mobilization for patients with chronic low back pain. The unpaired t test values have shown that there was significant difference between two groups in showing improvement in their quality of life in patients chronic low back ache with radiculopathy

\section{Discussion}

The result from statistical analysis of the present study showed that along with TENS with Traction, Mackenzie Technique is effective in decreasing pain, improving functional ability and increasing spinal extension in chronic low back pain with radiculopathy.

Within groups comparision showed significant reduction in VAS score, but between group analyses showed Mackenzie group showed more reduction in pain. The centralization effect of Mackenzie technique may contribute to reduction of pain. A study was done to find the effectiveness of McKenzie exercise in patients with lumbar radiculopathy.

A study was done to find out the effectiveness of McKenzie exercise in patient with lumbar radiculopathy. McKenzie group demonstrated significantly greater improvements in pain and function after three session.

Within group comparision showed significant reduction in MODQ scores, but between group analyses showed McKenzie group showed more reduction in MODQ scores. Lumbar spine range of motion, initially it was same for both groups, but after 4 weeks the McKenzie group scores better for spinal flexion, extension, side flexion. This study supports the use of McKenzie technique to increase the lumbar spine ROM and in reduction of pain.

This study supports the use of McKenzie technique to increase the lumbar spine ROM and in reduction of pain. Within groups comparsion showed significant increase in spinal extension ROM, but between group analyses showed McKenzie group showed more significant increase in spinal extension ROM.
The McKenzie technique is a more passive form of spinal manipulation in which patient produces the motion, position and forces that improve the condition. McKenzie back extension is a progression from lying prone to prone on hand with over pressure. These back extensions exercise from prone lying is assumed to have a greater effect in moving the disc content anterioly away from spinal nerves radicular symptoms of patient with derangement and also doing repeated extension exercise will help in maintaining and improving spinal extension.

\section{Limitations}

- Study focused only on Chronic Low Back Ache not on acute.

- Study was not focused on any particular occupation or athletic population

- The study has been conducted on small sized sample only.

- This study took shorter duration to complete.

- This study is not extended more than 4 weeks for a patient due to time constraint

\section{Recommendations}

- A similar study may be extended with larger sample.

- Future study should focus on other parameters like range of motion, ear of pain, muscular endurance and strength.

- Future studies should focus on particular group of occupations.

- Long term follow up should be made to find out the effect of the treatment.

\section{Summary and Conclusion}

From the result of this study through Mckenzie technique shows improvement in pain and range of motion in Chronic Low Back Ache. This technique has more advantages over functional abilities. Clinically it is important after Chronic LBA to regain the sufficient functional abilities.

Based on't' value and standard deviation, there is considerable improvement in the condition of the patient. Henceforth, Mckenzie technique help in improving quality of life of the patient.

Through the results, Alternative Hypothesis Is Accepted and also study could be concluded that there is a significant difference in Mckenzie technique patient with Chronic LBA.

\section{Reference}

1. Pinar Boreman. The Efficiencyb of Lambar Traction in the Management of patient with Low Back pain. Rheumatology. 2003; 23:82-86.

2. Elizabeth Smoots, Low Back pain. Bones joints and Muscles, 2008.

3. Van Tulder. conservative treatment of acute and chronic nonspecific low back pain: A systemiatic review of Randomized controlled Trials of most common intervention. Spine. 1997; 22:2128-2156.

4. Bart koesl W. diagnosis and treatment of sciatica. British Medical Journal, 2007; 337:1313-1317.

5. Brent Brotzman S, Kelvin Wilk E. Clinical Orthopedic Rehabilization 2nd edition, Mosby publications, 2003, 555-599.

6. Tarulli AW, Raynor EM. Lumbosacral radiculopathy. Neurology clinic. 2007; 25(2):387-405.

7. Anthony wheeler H. Pathophysiology of chronic back pain. Pain and orthopedics neurology, 2009.

8. Me Kenzie RA. Mechanical Diagnosis and therapy for 
Low Back Pain, Physical therapy for low back Churchill Livingstone New York, 1987, 157-174.

9. Aoron Sufka. Centralization of low back pain and perceived functional outcomes. JOSPT. 19821; 7(3):205212.

10. David Butler S. Mobilization of Nervous system Churchill Livingstone, 1996:185-199.

11. Fried. Transcutaneous Electrical Nerve Stimulation: Archives Physical medicine Rehabilitation. 1984; 65(5):228-231

12. Schenk. A historical review of manual therapy and the American Academy of Orthopaedics Manual Physical Therapist. Journal Manual Manipulative Therapy. 2000; 8(3): 138 .

13. Goldby. A randomized controlled trial comparing the McKenzie method of mechanical diagnosis and therapy with a non-prescriptive exercise regime in the conservative treatment of chronic low back pain. Proceedings $4^{\text {th }}$ McKenzie institute International Conference, Cambridge, England, 1995.

14. Nwuga, Nwuga. Relative therapeutic efficacy in back pain management. Physiotherapy Theory and Practice. 1985; 1(2):99-105.

15. Frtiz. Is patients with low back pain likely to benefit from Mechanical Traction? Result of a Randomized clinical Trial ad sub grouping analysis. Spine. 2007; 32(26):E793-E800.

16. David Broeder A. Effective of Extension Oriented treatment approach in a subgroup of patients with low back pain-a randomized clinical Trial. Physical therapy. 2007; 87(12):1608-1618.

17. Adams N. Psychophysiology and neurochemical sabstractes of chronic low back pain andmodulation by treatment. Physiotherapy. 1993; 79:86-91.

18. Demoulin C, Distrée V, Tomasella M, Crielaard J, Vanderthommen M. Lumbar functional instability: a critical appraisal of the literature. Annales de Réadaptationet de Médecine Physique. 2007; 50(8):677684.

19. Frih Z, Fendri Y, Jellad A, Boudoukhaneand S, Rejeb N. Efficacy and treatment compliance of a home-based rehabilitation programme for chronic low back pain: A randomized, controlled study. Annals of Physical and Rehabilitation Medicine. 2009; 52(6):485-496.

20. Tulder M, Koes B, Malmivaara A. Outcome of noninvasive treatment modalities on back pain: an evidencebased review. Eur. Spine J, 2006; 15:64-81.

21. Sharma M, Haas M, Stano M, Spegman A, Gehring R. Determinants of Costs and Pain Improvement for Medical and Chiropractic Care of Low Back Pain. Journal of Manipulative and Physiological Therapeutics. 2009; 32(4):252-261.

22. Bekkering G, Tulder M, Hendriks E. Implementation of clinical guidelines on physical therapy for patients with low back pain: randomized trial comparing patient outcomes after a standard and active implementation strategy. Phys Ther. 2005; 85:544-555.

23. Nafissi S, Niknam S, Hosseini S. Electrophysiological evaluation in lumbosacral radiculopathy. Ir $\mathrm{J}$ neurol. 2012; 11(3):83-86.

24. Wainner RS, Fritz JM, Irrgang JJ, Boninger ML, Delitto A, Allison S. Reliability and diagnostic accuracy of the clinical examination and patient self-report measures for cervical radiculopathy. Spine. 2003; 28(1):55-62.

25. Tarulli AW, Raynor EM. Lumbosacral radiculopathy. 